\title{
COUGH SOUND ANALYSIS
}

\section{Martinek J. ${ }^{1}$, Klco P. ${ }^{2}$, Vrabec M. ${ }^{1}$, Zatko T. ${ }^{1}$, Tatar M. ${ }^{1}$, Javorka M. ${ }^{2}$}

\author{
${ }^{1}$ Department of Pathological Physiology, Jessenius Faculty of Medicine in Martin, Comenius University, Slovak \\ Republic \\ ${ }^{2}$ Department of Physiology, Jessenius Faculty of Medicine in Martin, Comenius University, Slovak Republic
}

\begin{abstract}
Cough is the most common symptom of many respiratory diseases. Currently, no standardized methods exist for objective monitoring of cough, which could be commercially available and clinically acceptable. Our aim is to develop an algorithm which will be capable, according to the sound events analysis, to perform objective ambulatory and automated monitoring of frequency of cough. Because speech is the most common sound in 24-hour recordings, the first step for developing this algorithm is to distinguish between cough sound and speech. For this purpose we obtained recordings from 20 healthy volunteers. All subjects performed continuous reading of the text from the book with voluntary coughs at the indicated instants. The obtained sounds were analyzed using by linear and non-linear analysis in the time and frequency domain. We used the classification tree for the distinction between cough sound and speech. The median sensitivity was $100 \%$ and the median specificity was $95 \%$. In the next step we enlarged the analyzed sound events. Apart from cough sounds and speech the analyzed sounds were induced sneezing, voluntary throat and nasopharynx clearing, voluntary forced ventilation, laughing, voluntary snoring, eructation, nasal blowing and loud swallowing. The sound events were obtained from 32 healthy volunteers and for their analysis and classification we used the same algorithm as in previous study. The median sensitivity was $86 \%$ and median specificity was $91 \%$. In the final step, we tested the effectiveness of our developed algorithm for distinction between cough and non-cough sounds produced during normal daily activities in patients suffering from respiratory diseases. Our study group consisted from 9 patients suffering from respiratory diseases. The recording time was 5 hours. The number of coughs counted by our algorithm was compared with manual cough counts done by two skilled co-workers. We have found that the number of cough analyzed by our algorithm and manual counting, as well, were disproportionately different. For that reason we have used another methods for the distinction of cough sound from non-cough sounds. We have compared the classification tree and artificial neural networks. Median sensitivity was increasing from $28 \%$ (classification tree) to $82 \%$ (artificial neural network), while the median specificity was not changed significantly. We have enlarged our characteristic parameters of the Mel frequency cepstral coefficients, the weighted Euclidean distance and the first and second derivative in time. Likewise the modification of classification algorithm is under our interest.
\end{abstract}

Keywords: Cough sound, linear analysis, non-linear analysis, sensitivity, specificity

\section{INTRODUCTION}

Since the 1950, there has been an interest in making objective monitoring of cough frequency. The first published systems consisted of reel-to-reel tape recorder and the patients have to spend the entire recording time in the single room containing a microphone. The obtained cough sounds were counted manually by listening to the entire sound recordings $(1,2)$. The major problems with these systems were the laborious nature of the manual cough counting and the restriction of the patients in the single room. For these reasons these systems never became clinically acceptable. Recent development of recording systems, computer technology and memory cards permit to use the digital technology in the sound analysis and their classification. The cough sound events can be recorded using the digital voice recording systems and after the recording time can be analyzed using personal computer. The obtained sound events are classified according to the mathematical sound events analysis alone $(3,4)$, or the input data are enlarged to the EMG recordings with the aim to

Address for correspondence:

Jozef Martinek, Department of Pathological Physiology, Jessenius Faculty of Medicine, Comenius University, Sklabinska Str. 26, 03601 Martin, Slovakia. Phone: +421 432633 606, e-mail: martinek@jfmed.uniba.sk 
better distinguish the analyzed cough sounds from the other non-cough sounds $(5,6)$. The obtained sound events are analyzed in the time and frequency domain $(7,8,9,10)$. For sound events classification are used the classification trees (11), artificial neural networks (3), hidden Markov models (4), or a hybrid model, which is the combination of the artificial neural network and hidden Markov model (12).

Despite the rising interest to prepare an accurate algorithm for objective monitoring frequency of cough according to the sound events analysis, to date any standardized method which will be commercially available and clinically acceptable does not exist.

We are presenting an overview of the development of our algorithm for 24-hours objective monitoring frequency of cough in humans. The cough sounds are distinguished from the other non-cough sounds according to mathematical sound events analysis and are classified using classification tree or artificial neural network in the classification task. The development of our algorithm for objective monitoring frequency of cough was published in our recent studies $(11,13,14,16,17)$.

\section{MATERIAL AND METHODS}

\section{Subjects}

Firstly we obtained sound records from 20 healthy subjects. Our study group performed continuous reading of a text from a book with voluntary coughs (46 cough events) performed at the instants indicated in the text. The recording lasted about $20 \mathrm{~min}$. Before reading the text, the subjects coughed voluntarily three times to obtain their individual cough sound pattern (11).

Next study was focused on the other non-cough sounds produced from the upper airways. We obtained sound records from 32 healthy volunteers. The recorded sounds were: induced and voluntary coughs, induced sneezing, voluntary throat and nasopharynx clearing, voluntary forced ventilation, laughing, voluntary snoring, eructation, nasal blowing, loud swallowing and speech. The induced coughs were provoked by single-breath inhalation of capsaicin $(250 \mathrm{~mol} / \mathrm{l})$ and sneezing was induced by intranasal administration of histamine solution $(8 \mathrm{mg} / \mathrm{ml})(14)$.

In the last step we tested the accuracy of our developed algorithm for distinction between spontaneous cough and other non-cough sounds. We obtained sound records from 9 patients suffering from respiratory diseases. The patients were hospitalized at the Clinic of Tuberculosis and Respiratory Diseases, Jessenius Faculty of Medicine, Comenius University, Martin. The recording time was 5 hours (from 10:00 to 15:00). During the recording time the patients could perform their normal daily activities. At the beginning of the recording, the patients coughed voluntary three times to obtain their individual cough sound pattern (13).

\section{Recording system}

For sound events recording obtained from the healthy volunteers we used the system consisted of a portable digital voice recorder (Sony, ICD-MX20, Sony Corporation China) with the sampling frequency $7 \mathrm{kHz}$ in the long play mode and the miniature omnidirectional condenser microphone (ATR35s, Audio-Technica U.S., Philippines) with the frequency response between $50-18000 \mathrm{~Hz}$. The microphone was attached to the subject's chest and was covered by a plastic foam membrane to suppress sounds coming from the outer environment. The low sampling frequency of the used recorder allowed analyzing the acquired sound data only in the frequency range between $60-3500 \mathrm{~Hz}$. For this reason we changed our recording system to the new system consisted of the portable linear PCM recorder (PCM-M10, Sony Corp., China) and miniature condenser microphone (DPA 4061BM). The recorder permits to perform sound recordings with the sampling frequency of $44 \mathrm{kHz}$ and resolution 16 bits per sample, which is sufficient for detailed cough sound analysis. The changed microphone has 
better frequency characteristic. In the effort to better suppress sounds coming from the outer environment we tested new materials for acoustical equalizer construction. We used self developed acoustical equalizer to suppress sounds coming from the outer environment. This equalizer still undergoes an evolution.

\section{Processing of the obtained data}

The obtained sound records were transferred to the PC and further analyzed. The detection of the sound events and removing of the quite segments from the raw records was the first step in the sound analysis. The algorithm for sound events detection was described in our recently published studies $(11,13,14)$. For sound events detection we used the moving window which moved over the whole audio signal without overlap. For each position of the moving window was calculated the value of standard deviation (SD) and compared with empirically determined threshold value. The portions of the signal containing quite segments reached only small value of SD related to the inherent noise present in the signal. The portions of the signal containing sound events reached relatively high degree of SD which exceeded the threshold value. For sound events exclusion we used the length of each identified event. Because the mean duration of the voluntary cough sound acquired from healthy volunteers is $0.3 \pm 0.01 \mathrm{sec}$ (15), the detected sound events which were shorter as $0.1 \mathrm{sec}$ were excluded from further analysis. The detected sound events which were longer as $0.1 \mathrm{sec}$ were stored in separate WAV files.

\section{Sound events analysis}

The characteristic parameters of the cough sounds, which will be able to distinguish the cough event from the non-cough sounds, have been elaborated. The acquired sound events have been analyzed in the time and frequency domain. Firstly we measured the length of the each identified sound event (parameter length). From the Total power (TP) corresponding to the area under the power spectral density (PSD) curve we determined the values of the first local (parameter local) and global (parameter global) maximum and the time of their occurrence (parameters time $e_{\text {local }}$ and time global $_{\text {) }}$. We determined the mean and maximal values of the sound events intensity (parameters $T P_{\text {mean }}$ and $T P_{\max }$ ). The cough sound can be characterized by the sudden rise of a waveform with one or several large amplitudes, while voice sounds show a gradual rise of their waveform (10). For this reason the characteristic parameter, which can be calculated, was the parameter slope. Parameter slope was computed as a value of the first local maximum divided by the time of its occurrence. Next we found all local maxima and minima in the time course of the TP in sound events. We computed the parameter ratio of TPs of all local maxima divided by the sum of TPs of all local minima in a given sound events. From the 512 samples corresponding to the first local and global maxima we computed the values of sample entropy (parameters $S a m p E n_{\text {local }}$ and $S a m p E n_{\text {global }}$ ). From the frequency spectrum determined from these 512 samples we computed parameters skewness and kurtosis. Skewness is a measure of the asymmetry of the probability distribution of a real-valued random variable. Kurtosis is a measure of the "peakedness" of the probability distribution of a real-valued random variable.

Because these variables were insufficient for accurate distinction between spontaneous cough sounds and non-cough sounds acquired from patients with respiratory diseases we enlarged our characteristic parameters to the other parameters such as Mel frequency cepstral coefficients (MFCC), the values of the first and second derivative in time and the weighted Euclidean distance. The MFCC are frequently used in the speech recognition algorithms. They represent the short-term power spectrum of analyzed sound. The first and second derivative in time is used in order to better reflect the dynamic changes of the analyzed variable. The weighted Euclidean distance was computed as a measure of the distance between MFCC computed from three cough sound patterns obtained from each analyzed patient and the values of MFCC computed from the other analyzed sound events. 


\section{Sound events classification}

For sound events classification to the cough and non-cough sound groups we used the classification tree. This algorithm was successfully employed for distinction between voluntary coughs and other non-cough sounds acquired from healthy patients $(11,14)$. For distinction between spontaneous cough sounds from the other non-cough sounds acquired from patients with respiratory diseases we reached low value of accuracy (13). For this reason we changed our algorithm and used the artificial neural network in the classification task $(16,17)$. The artificial neural network is a mathematical model inspired by biological neural networks. For sound events classification we used the feedforward backpropagation neural network. The artificial neural network was trained according to the LevenbergMarquard algorithm on set of $50 \%$ from all non-cough sounds and on set of $10 \%$ from all cough sounds.

\section{RESULTS}

We reached good accuracy for distinction between voluntary cough sounds and speech obtained from healthy subjects (sensitivity was 100\%, specificity was 95\%) (11). These results were reached using classification tree in the classification task. The measured characteristic parameters were: the length of the entire identified sound event, the mean and maximal value of the sound event intensity, the value of the first local and global maximum and the time of their occurrence, parameter slope, parameter ratio, sample entropy and the values of skewness and kurtosis. All characteristic parameters were acquired using analysis in the time and frequency.

Relatively good accuracy (sensitivity $86 \%$, specificity 91\%) was reached for distinction between coughs and other non-cough sounds produced from the upper airways by healthy volunteers (14). The cough sound events $(n=152)$ included voluntary $(n=52)$, spontaneous $(n=8)$ and induced $(n=92)$ coughs. The non-cough sounds $(n=1554)$ were voluntary throat $(n=62)$ and nasopharynx clearing $(n=23)$, voluntary forced ventilation $(n=19)$, laughing $(n=125)$, voluntary snoring $(n=26)$, eructation $(n=18)$, nasal blowing $(n=85)$, induced sneezing $(n=30)$, speech $(n=1073)$ and other sounds $(n=93)$. From the total number of the 152 cough sounds were by our algorithm correctly classified 131 cough sounds. Remaining coughs were classified as non-cough sound events.

In the last step we tested the accuracy of our developed algorithm for distinction between spontaneous cough sounds and the other non-cough sounds acquired from the patients suffering from respiratory diseases. The accuracy of our developed algorithm was compared with manual cough counts obtained from two skilled co-workers. In this classification task we reached relatively low accuracy (sensitivity $28 \%$, specificity $99 \%$ ) (13). For this reason we enlarged the characteristic parameters to the MFCC, first and second derivative in time and the weighted Euclidean distance. We compared the accuracy of the classification tree and artificial neural network in the classification task (Tab 1). From our results we can see, that the artificial neural networks are more sensitive for classification task compared with clas-

Table 1: The classification accuracy for distinction between spontaneous cough sounds and other noncough sounds acquired from patients with respiratory diseases. The obtained sound events are classified using by classification tree or artificial neural network. From the table we can see, that the artificial neural network is more sensitive for classification compared with classification tree.

\begin{tabular}{|l|c|c|}
\hline Classification algorithm & sensitivity [\%] & specificity [\%] \\
\hline Classification tree & 28 & 99 \\
\hline Artificial neural network & 82 & 96 \\
\hline
\end{tabular}


sification tree $(16,17)$. Using by the classification tree in the classification task were from the number of 1074 cough sounds correctly classified only 300 cough sound events. The artificial neural networks caused that the number of properly classified coughs raised from the 300 coughs to 880 cough sound events. Remaining cough sounds were incorrect, classified as non-cough sounds.

\section{DISCUSSION}

This paper includes an overview of developing a mathematical algorithm for objective monitoring of the frequency of cough during 24-hours in ambulatory settings. In the first step we prepared a system for 24-hours recording of the cough sound and method for suppressing sounds coming from the outer environment. The environmental sounds were suppressed using self developed acoustical equalizer.

The characteristic parameters of the cough sound were acquired due to an analysis of the voluntary coughs obtained from the healthy volunteers. We assume that the speech is the most common sound present in the normal daily patient's activity. For that reason was our first study aimed to distinction between voluntary cough sound and speech (11). In the next step we enlarged the analyzed sound events to the other non-cough sounds produced from the upper airways (14). The recorded sounds were analyzed in the time and frequency and classified using the classification tree. Our results are comparable with the study of Vizel et al. (18). In this study the authors recorded voluntary cough sounds from healthy volunteers while laying supine, sitting, sitting with strong ambient noise, walking and climbing stairs. Each phase lasted 5 minutes in which the subjects did not coughed for 2 minutes, then voluntary coughed for 2 minutes, then performed voluntary coughs of graded intensity, throat clears and talked (counting from one to ten) for 1 minute. The total length of the recordings was 25 minutes per subject. Authors reached the overall specificity $94 \%$ and the overall sensitivity $96 \%$. In this study there were the cough "candidates" identified based on their energy characteristics and cough amplitude pattern previously established from voluntary and spontaneous coughs. The „candidates“ were verified based on their fit to a cough pattern in both the time and frequency. It is demanding to compare our results with other studies, because the measurement protocol is not similar. Many authors compare spontaneous cough sounds in patients with respiratory diseases with other non-cough sounds produced by their normal daily activities $(3,4)$. We compared the voluntary cough sounds vs. other non-cough sounds produced from the upper airways.

In the last step we aimed for distinction between spontaneous cough sounds and noncough sounds produced by the patients with respiratory diseases. Firstly, the obtained sound records were analyzed and classified using the same algorithm as in previous study (time and frequency, classification tree). The median sensitivity was $28 \%$ and median specificity was $99 \%$. The low classification accuracy is caused by the wide variability of the spontaneous cough sounds in their frequency spectrum. This variability is typical not only between the individual patients, but also in the same patient during the 24-hours. In effort to reach higher accuracy for distinction between cough and non-cough sounds produced by patients suffering from respiratory diseases we used MFCC (Mel frequency cepstral coefficients) and the first and second derivative in the time. In the classification step we used the artificial neural networks. The median sensitivity was increased from $28 \%$ (classification tree) to $82 \%$ (artificial neural network). The value of median sensitivity was not changed significantly. We proved that the artificial neural networks are more sensitive for sound events classification compared with classification trees. The accuracy of our developed algorithm is comparable with recently published studies $(3,4)$. For the future we try to improve the accomplishment of our developed algorithm. We want to better describe the time changes of the characteristic parameters of the cough sound. For better distinction between cough and non-cough sounds we want to adjust the artificial neural networks to the conditions of spontaneous cough sound obtained from patients suffering from respiratory diseases. 


\section{REFERENCES}

1. Woolf $\mathrm{CR}$, Rosenberg A. Objective assessment of cough suppressants under clinical conditions using a tape recorder system. Thorax 1964; 19: 125-30.

2. Loudon RG, Brown LC. Cough frequency in patients with respiratory disease. Am Rev Respir Dis 1967; 96(6): 1137-43.

3. Barry SJ, Dane AD, Morice AH, Walmsley AD. The automatic recognition and counting of cough. Cough 2006; 2: 8 , doi 10.1186/1745-9974-2-8.

4. Matos S, Birring SS, Pavord ID, Evans DH. Detection of cough signals in continuous audio recordings using hidden Markov models. IEEE Trans Biomed Eng 2006; 53(6): 1078-83.

5. Hsu JY, Stone RA, Logan-Sinclair RB, Worsdell M, Busst CM, Chung KF. Coughing frequency in patients with persistent cough: assessment using a 24 hour ambulatory recorder. Eur Respir J 1994; 7(7): 1246-53.

6. Chang AB, Newman RG, Phelan PD, Robertson CF. A new use for an old Holter monitor: an ambulatory cough meter. Eur Respir J 1997; 10(7): 1637-9.

7. Murata A, Taniguchi Y, Hashimoto Y, Kaneko Y, Takasaki Y, Kudoh S. Discrimination of productive and nonproductive cough by sound analysis. Intern Med 1998; 37(9): 732-5.

8. Olia PM, Sestini P, Vagliasindi M. Acoustic parameters of voluntary cough in healthy non-smoking subjects. Respirology 2000; 5(3): 271-5.

9. Van Hirtum A, Berckmans D. Automated recognition of spontaneous versus voluntary cough. Med Eng Phys 2002; 24(7-8): 541-510. Murata A, Ohota N, Shibuya A, Ono H, Kudoh S. New non-invasive automatic cough counting program based on 6 types of classified cough sounds. Intern Med 2006; 45(6): 391-7.

10. Murata A, Ohota N, Shibuya A, Ono H, Kudoh S. New non-invasive automatic cough counting program based on 6 types of classified cough sounds. Intern Med 2006; 45(6):391-7.

11. Martinek J, Tatar M, Javorka M. Distinction between voluntary cough sound and speech in volunteers by spectral and complexity analysis. J Physiol Pharmacol 2008; 59(6): 433-40.

12. Shin SH, Hashimoto T, Hatano S. Automatic detection system for cough sounds as a symptom of abnormal health condition. IEEE Trans Inf Technol Biomed 2009; 13(4): 486-93.

13. Martinek J, Bencova A, Tatar M, Vrabec M, Zatko T, Javorka M. Examination of cough and non-cough sounds by spectral and complexity analysis in patients suffering from respiratory diseases. Acta Med Mart 2009; 9(3): 12-17.

14. Martinek J, Zatko T, Tatar M, Javotka M. Distinction of cough from other sounds produced by daily activities in the upper airways. Bratisl Lek Listy 2011; 112(3): 120-4.

15. Korpas J, Sadlonova J, Vrabec M. Analysis of the cough sound: an overview. Pulm Pharmacol 1996; 9(5-6): 261-8.

16. Klco P, Martinek J, Tatar M, Javorka M. Application of the artificial neural networks for the cough sound classification (in Slovak). New knowledge in respirology (the lectures in XXII. Martin's Day of Breathing).

17. Martinek J, Tatar M, Javorka M. An Adjusted algorithm for cough sound analysis in patients with respiratory diseases (in Slovak). New knowledges in respirology (the lectures in XXII. Martin's Day of Breathing).

18. Vizel E, Yigla M, Goryachev Y, Dekel E, Felis V, Levi H, Kroin I, Godfrey S, Gavriely N. Validation of an ambulatoty cough detection and counting application using voluntary cough under different conditions. Cough 2010; $6: 3$.

\section{Acknowledgement:}

This study was supported by European grant OPV - 26110230031/04/PD/2010

Received: January,10,2013

Accepted: February,7,2013 